\title{
Shape functions for high-order shear deformation beam element
}

\author{
Himanshu Gaur $^{1}{ }^{\&} 2$, Mahmoud Dawood ${ }^{1} \&$ Ram Kishore Manchiryal $^{1}$ \\ Department of Civil Engineering, Middle East College, Muscat, Oman. \\ Institute of Structural Mechanics, Bauhaus-Universitat Weimar, Marienstrasse 15, D-99423 \\ Weimar, Germany.
}

\begin{abstract}
In this article, shape functions for higher-order shear deformation beam theory are derived. For the two nodded beam element, transverse deflection is assumed as cubic polynomial. By using equations of equilibrium of high-order theory that are already derived by J. N. Reddy in 1997, equation for slope of high- order theory is found. Finally with the boundary conditions of beam element and assumed kinematics of high-order theory, shape functions are derived.
\end{abstract}

Key Words: High-order shear deformation theory, shape functions, finite element analysis.

\section{Introduction:}

The coordinate system adopted in this article are represented in figure -1. In this $\mathrm{x}$ - axis is along the longitudinal axis of the beam and $y-$ and $z$ - axis are along the two transverse directions as shown. Deformations in $\mathrm{x}, \mathrm{y}$ and $\mathrm{z}$ directions are represented as $\mathrm{u}, \mathrm{v}$ and $\mathrm{w}$ respectively.
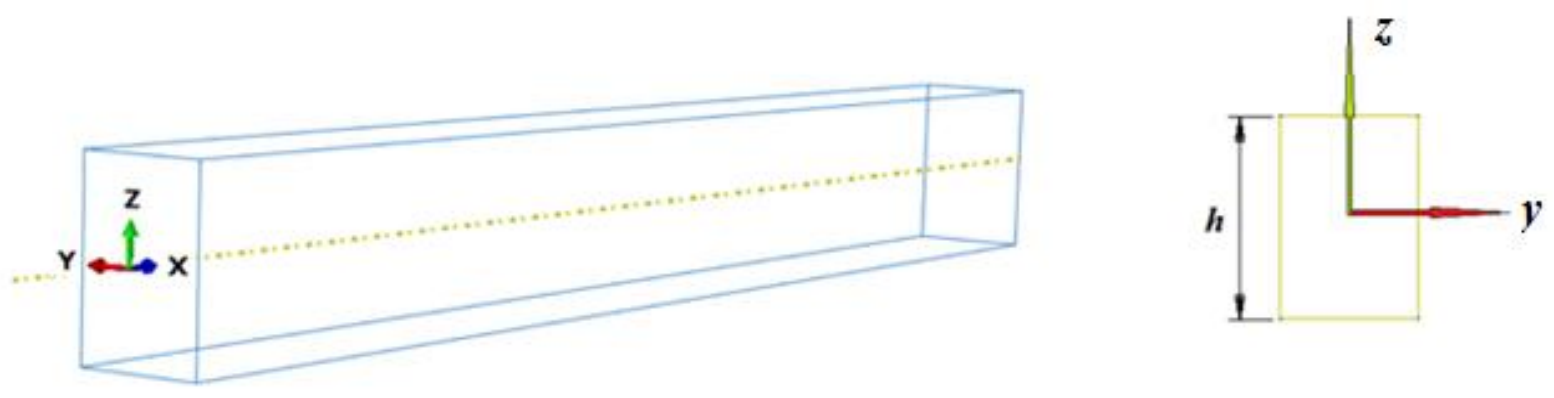

Figure -1: Beam in the Cartesian coordinate system.

One of the earliest development in the history of shape functions for structural element is Hermitian Shape functions (G. P. Bazeley et al. 1965) in which transverse displacement is interpolated from nodal rotations as well as nodal displacements. Hermitian Shape functions are $C^{1}$ type functions which makes both deflection $w$ as well as slope $\frac{d w}{d x}$ continuous in between the elements. For structural elements, Hermitian interpolations are indeed derived with the EulerBernoulli beam theory (Seon M. Han et. al. 2007). Euler-Bernoulli beam theory is one of the 
oldest and one of the simplest beam theory presented so far which was given by Leonhard Euler and Daniel Bernoulli circa 1750. It was widely accepted in the late $19^{\text {th }}$ century. According to the Euler-Bernoulli beam theory displacement field in xz- plane is defined as

$$
\begin{aligned}
& U(x, z)=u-z \frac{d w_{0}}{d x} \\
& W(x, z)=w_{0}(x)
\end{aligned}
$$

In which $w_{0}$ is the transverse deflection at the neutral axis (i.e. $\mathrm{z}=0$ ) of the beam. According to this assumption, a plane which was normal to the neutral axis before deformation remains plane and remains normal to the neutral axis after deformation. And hence, according to this theory, rotation of any section along the beam axis can be directly calculated by simple differentiation of lateral deformation of the neutral axis $\left(w_{0}\right)$ with respect to $x$. This Euler- Bernoulli assumption totally forbids transverse shear deformation component $-\mathrm{du} / \mathrm{dz}$ and hence can be regarded as the case of pure bending.

Further development in the beam theories is made by Timoshenko et al. in 1921 and 1970 and is called as Timoshenko beam theory (TBT). The kinematic assumptions in this beam theory in xzplane can be represented as.

$$
\begin{aligned}
& U(x, z)=u-z\left(\frac{d w_{0}}{d x}+\chi\right) \\
& W(x, y)=w_{0}(x)
\end{aligned}
$$

Here ' $\chi$ ' can be regarded as transverse shear deformation component -du/dz. It is assumed to be constant $(\chi)$ along the depth of the beam according to this theory. This shear deformation is also assumed to be constant along the length of the beam. From the strength of material theory as well as from the theory of elasticity (S. P. Timoshenko et al. 2010), beam section not only rotates as well as warp along the transverse direction. Shear deformation is in-fact cubic in nature along the depth of the beam. Hence TBT requires 'shear correction factor' which is obtained by simple balance of shear strain energy by the constant shear assumption to that of the real cubic shear deformation across the depth of the cross-section (Klaus-Jürgen Bathe 2014). Shear correction factor depends upon the geometrical sectional properties of the beam. In 2003, A. Bazoune et al. derived shape functions for this theory.

Further development in the beam theory is High-order beam theory (HBT). Which not only considers transverse shear deformation component but also considers warping of the cross section Jemielita 1975, Bickford 1982, Levinson 1981 and Reddy 1997. It captures true quadratic variation of shear strains along the section depth and there is no need to use shear correction factor. It gives more realistic picture of shear deformation in beam theory. Displacement field in this theory in xz- plane is represented as.

$$
U(x, z)=u+z\left(\Phi(x)-\alpha z^{2}\left(\Phi(x)+\frac{d w_{0}}{d x}\right)\right)
$$




$$
W(x, z)=w_{0}(x)
$$

Here ' $\alpha$ ' is the constant value of which is $\frac{4}{3 h^{2}}$ with ' $h$ ' being the depth of the cross section. $\Phi(x)$ and $w_{0}$ represents the slope $\left(\frac{d u_{0}}{d z}\right)$ and lateral deformation respectively at the neutral surface $\mathrm{z}=$ 0 . This is also called Third-order beam theory. Third-order theory is sufficient and accurate enough to get the boundary conditions of zero shear stress at the top and bottom surfaces of the beam in the beam kinematics. Theories higher than third order is not required. Shape functions for Higher -order shear deformation theory are not derived so far in the literature. The objective of this study is to fill this gap.

\section{Importance of refined theories:}

Shear deformation is more pronounced in thick beams rather than slender beams. Studying shear deformation is crucial for the thick beams in getting real bending deformation of the thick beams. These effects are neglected in EBT. In order to describe the correct bending behavior of thick beams including shear deformation and the associated cross-sectional warping, shear deformation theories are required. This is done by selecting proper kinematics of the beam bending. To accomplish this, functions $f(z)$ is included in the displacement field of higher order theories. Few of the functions that are derived so far in the literature are as follows:

\begin{tabular}{|c|l|c|}
\hline S.N. & \multicolumn{1}{|c|}{ Author } & Function $f(z)$ \\
\hline 1 & S. A Ambartsumyan, 1958 & $f(z)=\left[\frac{z}{2}\left(\frac{h^{2}}{4}-\frac{z^{2}}{3}\right)\right]$ \\
\hline 2 & E. T. Kruszewski, 1909 & $f(z)=\left[\frac{5 z}{4}\left(1-\frac{4 z^{2}}{3 h^{2}}\right)\right]$ \\
\hline 3 & J. N. Reddy 1990 & $f(z)=z\left[1-\frac{4}{3}\left(\frac{z}{h}\right)^{2}\right]$ \\
\hline 4 & M. Touratier, 1991 & $f(z)=\frac{h}{\pi} \operatorname{Sin} \frac{\pi z}{h}$ \\
\hline 5 & K. P. Soldatos 1992 & $f(z)=\left[z \operatorname{Cosh}\left(\frac{1}{2}\right)-h \operatorname{Sinh}\left(\frac{z}{h}\right)\right]$ \\
\hline 6 & M. Karama, et. al. 2003 & $f(z)=z \exp \left[-2\left(\frac{z}{h}\right)^{2}\right]$ \\
\hline 7 & S. S. Akavci, 2007 & $f(z)=\frac{3 \pi}{2}\left[h \tanh \left(\frac{z}{h}\right)-z \sec ^{2} h\left(\frac{1}{2}\right)\right]$ \\
\hline
\end{tabular}

In this article, we will use function $f(z)$ derived by J. N. Reddy in 1990.

\section{Finite Element Discretization:}

Consider any two- node beam element of length $l$ in plane bending (xz- plane) where each node has three degrees of freedom. The nodal displacement vector $\{e\}$ can be defined by the element degrees of freedom by.

$$
\{e\}_{6 \times 1}=\left[\begin{array}{llllll}
u_{1} & w_{1} & \theta_{1} & u_{2} & w_{2} & \theta_{1}
\end{array}\right]^{T}
$$


Here $u_{1}$ and $u_{2}$ are axial displacements of the two nodes in $\mathrm{x}-$ direction, $w_{1}$ and $w_{2}$ are lateral displacements and $\theta_{1}$ and $\theta_{2}$ are rotations of the two nodes in xz- plane.

According to the standard finite element procedure, the elastic deformation of any arbitrary point of the beam section can be expressed as

$$
\{d\}=[\mathcal{N}]\{e\}
$$

Where $\{d\}$ is elastic deformation vector of beam element and $[\mathcal{N}]$ is the matrix of shape functions used to model the deformations. In this study, shape functions for axial deformation is considered as linear.

\section{Equations for third order beam theory:}

Axial and shear strain in third order beam theory can be derived from the displacement assumptions of equation - (3) as follows.

$$
\begin{aligned}
& \varepsilon_{x x}=\frac{\partial U}{\partial x}=z \frac{d \Phi}{d x}-\alpha z^{3}\left(\frac{d \Phi}{d x}+\frac{d^{2} w_{0}}{d x^{2}}\right) \\
& \gamma_{x z}=\frac{\partial W}{\partial x}+\frac{\partial U}{\partial z}=\frac{d w_{0}}{d x}+\Phi-\beta z^{2}\left(\Phi+\frac{d w_{0}}{d x}\right)
\end{aligned}
$$

Where

$$
\alpha=\frac{4}{3 h^{2}}, \quad \beta=3 \alpha=\frac{4}{h^{2}}
$$

The equations of equilibrium in High order theory can be written as (Reddy 1997).

$$
\begin{aligned}
& \frac{d M_{x x}}{d x}-\alpha \frac{d P_{x}}{d x}-Q_{x}+\beta R_{x}=0 \\
& \frac{d Q_{x}}{d x}-\beta \frac{d R_{x}}{d x}+\alpha \frac{d^{2} P_{x x}}{d x^{2}}+q=0
\end{aligned}
$$

Where $P_{x x}$ and $R_{x}$ are higher order stress resultants. Stress resultants $\left(M_{x x}, P_{x x}, Q_{x}\right.$ and $\left.R_{x}\right)$ can be related to the strains by the relations.

$$
\begin{aligned}
& M_{x x}=D_{x x} \frac{d \Phi}{d x}-\alpha F_{x x}\left(\frac{d \Phi}{d x}+\frac{d^{2} w_{0}}{d x^{2}}\right) \\
& Q_{x}=\left(A_{x z}-\beta D_{x z}\right)\left(\Phi+\frac{d w_{0}}{d x}\right) \\
& P_{x x}=F_{x x} \frac{d \Phi}{d x}-\alpha H_{x x}\left(\frac{d \Phi}{d x}+\frac{d^{2} w_{0}}{d x^{2}}\right) \\
& R_{x}=\left(D_{x z}-\beta F_{x z}\right)\left(\Phi+\frac{d w_{0}}{d x}\right)
\end{aligned}
$$

Where $D_{x x}$ is the bending stiffness, $A_{x x}$ is the shear stiffness and $F_{x x}, H_{x x}, D_{x z}$ and $F_{x z}$ are higher order stiffnesses which can be further expressed as. 


$$
\begin{aligned}
D_{x x}=\int_{A} E_{x} z^{2} d A & =E_{x} I_{x x}^{(2)}, & F_{x x}=\int_{A} E_{x} z^{4} d A & =E_{x} I_{y y}^{(4)} \\
H_{x x}=\int_{A} E_{x} z^{6} d A & =E_{x} I_{y y}^{(6)}, & A_{x z}=\int_{A} G_{x z} d A & =G_{x z} A \\
D_{x z}=\int_{A} G_{x z} z^{2} d A & =G_{x z} I_{y y}^{(2)}, & F_{x z}=\int_{A} G_{x z} z^{4} d A & =G_{x z} I_{y y}^{(4)}
\end{aligned}
$$

Here A is cross sectional area of beam. $E_{x}$ and $G_{x z}$ are Young's modulus and shear modulus respectively and $I_{y y}^{(i)}$ denotes the $i$-th area moment of inertia about the $y$ - axis:

$$
I_{y y}^{(i)}=\int_{A}(z)^{i} d A
$$

\section{The displacement field:}

The kinematic relation for the in-plane beam deformation (xz- plane) can be given by the equation - (3). In beam bending in xz-plane (figure-1), total vertical deformation will comprise of because of bending $w_{b}$ and because of transverse shear $w_{s}$ (S. P. Timoshenko 1921).

$$
w=w_{b}+w_{s}
$$

The relationship between total slope, bending rotation and transverse shear can be written as

$$
\frac{\partial w}{\partial x}=\frac{\partial w_{b}}{\partial x}+\frac{\partial w_{s}}{\partial x}
$$

\section{Derivation of shape functions:}

Let us consider the two nodded beam element. Since there are four nodal degrees of freedom at the two nodes, we assume a cubic polynomial. Hence the transverse deformation $W(x)$ at any arbitrary location along the axis can be expressed as:

$$
W(x)=a_{0}+a_{1} x+a_{2} x^{2}+a_{3} x^{3}
$$

The slope due to bending can be obtained by

$$
\frac{\partial w}{\partial x}=\frac{\partial w_{b}}{\partial x}+\frac{\partial w_{s}}{\partial x}=\Phi-\alpha z^{2}\left(\Phi+\frac{d w_{0}}{d x}\right)
$$

Equation - (15) gives the slope at any arbitrary section of the beam length and this should be equal to the differentiation of the assumed transverse deformation - (14).

$$
\frac{\partial w}{\partial x}=a_{1}+2 a_{2} x+3 a_{3} x^{2}=\Phi-\alpha z^{2}\left(\Phi+\frac{d w_{0}}{d x}\right)
$$

Differentiating again

$$
2 a_{2}+6 a_{3} x=\frac{d \Phi}{d x}-\alpha z^{2}\left(\frac{d \Phi}{d x}+\frac{d^{2} w_{0}}{d x^{2}}\right)
$$

Multiply throughout by $E_{x} z^{2}$ and integrating with respect to area of cross-section

$$
\left(2 a_{2}+6 a_{3} x\right) \int_{A} E_{x} z^{2} d A=\int_{A} E_{x} z^{2} d A \frac{d \Phi}{d x}-\alpha \int_{A} E_{x} z^{4} d A\left(\frac{d \Phi}{d x}+\frac{d^{2} w_{0}}{d x^{2}}\right)
$$




$$
\left(2 a_{2}+6 a_{3} x\right) E_{x} I_{y y}^{(2)}=D_{x x} \frac{d \Phi}{d x}-\alpha F_{x x}\left(\frac{d \Phi}{d x}+\frac{d^{2} w_{0}}{d x^{2}}\right)=M_{x x}
$$

Multiply throughout equation - (17) again by $E_{x} z^{4}$ and integrating with respect to area of crosssection

$$
\begin{aligned}
& \left(2 a_{2}+6 a_{3} x\right) \int_{A} E_{x} z^{4} d A=\int_{A} E_{x} z^{4} d A \frac{d \Phi}{d x}-\alpha \int_{A} E_{x} z^{6} d A\left(\frac{d \Phi}{d x}+\frac{d^{2} w_{0}}{d x^{2}}\right) \\
& \left(2 a_{2}+6 a_{3} x\right) E_{x} I_{y y}^{(4)}=F_{x x} \frac{d \Phi}{d x}-\alpha H_{x x}\left(\frac{d \Phi}{d x}+\frac{d^{2} w_{0}}{d x^{2}}\right)=P_{x x}
\end{aligned}
$$

Differentiating equation - (18) and (19) again with respect to $\mathrm{x}$ and substituting in equilibrium equation- (6).

$$
6 a_{3} E_{x} I_{y y}^{(2)}-\alpha 6 a_{3} E_{x} I_{y y}^{(4)}-\left(A_{x z}-\beta D_{x z}\right)\left(\Phi+\frac{d w_{0}}{d x}\right)+\beta\left(\left(D_{x z}-\beta F_{x z}\right)\left(\Phi+\frac{d w_{0}}{d x}\right)\right)=0
$$

Rearranging,

$$
6 a_{3} E_{x}\left(I_{y y}^{(2)}-\alpha I_{y y}^{(4)}\right)=A G_{x z}\left(\Phi+\frac{d w_{0}}{d x}\right)-2 \beta G_{x z} I_{y y}^{(2)}\left(\Phi+\frac{d w_{0}}{d x}\right)+\beta^{2} G_{x z} I_{y y}^{(4)}\left(\Phi+\frac{d w_{0}}{d x}\right)
$$

Or,

$$
6 a_{3} E_{x}\left(I_{y y}^{(2)}-\alpha I_{y y}^{(4)}\right)=\Phi\left(A G_{x z}-2 \beta G_{x z} I_{y y}^{(2)}+\beta^{2} G_{x z} I_{y y}^{(4)}\right)+\frac{d w_{0}}{d x}\left(A G_{x z}-2 \beta G_{x z} I_{y y}^{(2)}+\beta^{2} G_{x z} I_{y y}^{(4)}\right)
$$

With this expression slope $\Phi$ or $\frac{d u_{0}}{d z}$ at the neutral axis $(\mathrm{z}=0)$ can be represented in terms of beam lateral deflection $w_{0}$ as follows.

$$
\frac{6 a_{3} E_{x}\left(I_{y y}^{(2)}-\alpha I_{y y}^{(4)}\right)-\frac{d w_{0}}{d x}\left(A G_{x z}-2 \beta G_{x z} I_{y y}^{(2)}+\beta^{2} G_{x z} I_{y y}^{(4)}\right)}{\left(A G_{x z}-2 \beta G_{x z} I_{y y}^{(2)}+\beta^{2} G_{x z} I_{y y}^{(4)}\right)}=\Phi
$$

Or,

$$
\frac{6 a_{3} E_{x}\left(I_{y y}^{(2)}-\alpha I_{y y}^{(4)}\right)}{A G_{x z}-2 \beta G_{x z} I_{y y}^{(2)}+\beta^{2} G_{x z} I_{y y}^{(4)}}-\frac{d w_{0}}{d x}=\Phi
$$

Substituting $\Phi$ in equation - (16)

$$
\begin{gathered}
a_{1}+2 a_{2} x+3 a_{3} x^{2}=\Phi\left(1-\alpha z^{2}\right)-\alpha z^{2} \frac{d w_{0}}{d x} \\
a_{1}+2 a_{2} x+3 a_{3} x^{2}=\left(\frac{6 a_{3} E_{x}\left(I_{y y}^{(2)}-\alpha I_{y y}^{(4)}\right)}{A G_{x z}-2 \beta G_{x z} I_{y y}^{(2)}+\beta^{2} G_{x z} I_{y y}^{(4)}}-\frac{d w_{0}}{d x}\right)\left(1-\alpha z^{2}\right)-\alpha z^{2} \frac{d w_{0}}{d x}
\end{gathered}
$$

Or, 


$$
a_{1}+2 a_{2} x+3 a_{3} x^{2}=\frac{6 a_{3} E_{x}\left(I_{y y}^{(2)}-\alpha I_{y y}^{(4)}\right)\left(1-\alpha z^{2}\right)}{\left(A G_{x z}-2 \beta G_{x z} I_{y y}^{(2)}+\beta^{2} G_{x z} I_{y y}^{(4)}\right)}-\frac{d w_{0}}{d x}
$$

Or

$$
\frac{d w_{0}}{d x}=a_{1}+2 a_{2} x+3 a_{3} x^{2}-\frac{6 a_{3} E_{x}\left(I_{y y}^{(2)}-\alpha I_{y y}^{(4)}\right)\left(1-\alpha z^{2}\right)}{\left(A G_{x z}-2 \beta G_{x z} I_{y y}^{(2)}+\beta^{2} G_{x z} I_{y y}^{(4)}\right)}
$$

or

$$
\frac{d w_{0}}{d x}=a_{1}+2 a_{2} x+3 a_{3} x^{2}-\frac{6 a_{3} E_{x}\left(I_{y y}^{(2)}-\alpha I_{y y}^{(4)}\right)^{2}}{\left(A G_{x z}-2 \beta G_{x z} I_{y y}^{(2)}+\beta^{2} G_{x z} I_{y y}^{(4)}\right)}
$$

Let us say

$$
\frac{E_{x}\left(I_{y y}^{(2)}-\alpha I_{y y}^{(4)}\right)^{2}}{\left(A G_{x z}-2 \beta G_{x z} I_{y y}^{(2)}+\beta^{2} G_{x z} I_{y y}^{(4)}\right)}=\beta
$$

Hence

$$
\frac{d w_{0}}{d x}=a_{1}+2 a_{2} x+a_{3}\left(3 x^{2}-6 ß\right)
$$

Once getting expressions for deflection $w_{0}$ and slope at the neutral axis $\frac{d w_{0}}{d x}$, shape functions can easily be found by applying the following boundary conditions for beam deformations.

$$
\begin{aligned}
w(0)=w_{1} \quad \text { and } & w(l)=w_{2} \\
\left.\frac{d w_{0}}{d x}\right|_{x=0}=\theta_{1} \quad \text { and } & \left.\frac{d w_{0}}{d x}\right|_{x=l}=\theta_{2}
\end{aligned}
$$

Substituting,

$$
\begin{aligned}
& w_{1}=a_{0} \\
& \theta_{1}=a_{1}+6 ß a_{3} \\
& w_{2}=a_{0}+a_{1} l+a_{2} l^{2}+a_{3} l^{3} \\
& \theta_{2}=a_{1}+2 a_{2} l+a_{3}\left(3 l^{2}-6 ß\right)
\end{aligned}
$$

In matrix form

$$
\left\{\begin{array}{l}
w_{1} \\
\theta_{1} \\
w_{2} \\
\theta_{2}
\end{array}\right\}=\left[\begin{array}{lllr}
1 & 0 & 0 & 0 \\
0 & 1 & 6 ß & 0 \\
1 & l & l^{2} & l^{3} \\
0 & 1 & l & 3 l^{2}-6 ß
\end{array}\right]\left\{\begin{array}{l}
a_{0} \\
a_{1} \\
a_{2} \\
a_{3}
\end{array}\right\}
$$

In the more compact form 


$$
\{d\}=[A]\left\{a_{i}\right\}
$$

From which

$$
\left\{a_{i}\right\}=[A]^{-1}\{d\}
$$

Solving for $\left\{a_{i}\right\}$

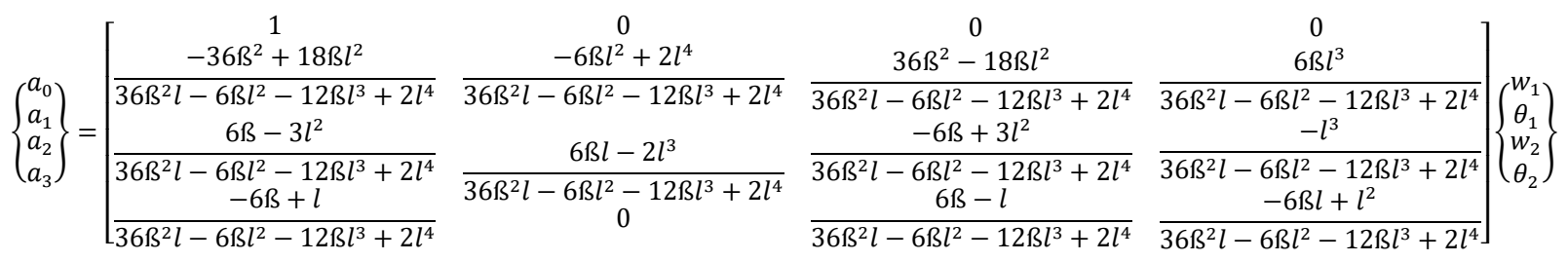

Let us say

$$
\begin{gathered}
36 \Omega^{2} l-6 ß l^{2}-12 \beta l^{3}+2 l^{4}=\mho \\
\left\{\begin{array}{l}
a_{0} \\
a_{1} \\
a_{2} \\
a_{3}
\end{array}\right\}=\left[\begin{array}{cccc}
\frac{1}{-36 \S^{2}+18 ß l^{2}} & \frac{-6 ß l^{2}+2 l^{4}}{\mho} & \frac{36 \beta^{2}-18 ß l^{2}}{\mho} & \frac{6 ß l^{3}}{\mho} \\
\frac{6 ß-3 l^{2}}{\mho} & \frac{6 ß l-2 l^{3}}{\mho} & \frac{-6 ß+3 l^{2}}{\mho} & \frac{-l^{3}}{\mho} \\
\frac{-6 ß+l}{\mho} & 0 & \frac{6 ß-l}{\mho} & \frac{-6 ß l+l^{2}}{\mho}
\end{array}\right]\left\{\begin{array}{c}
w_{1} \\
\theta_{1} \\
w_{2} \\
\theta_{2}
\end{array}\right\}
\end{gathered}
$$

Hence

$$
\begin{aligned}
& a_{0}=w_{1} \\
& a_{1}=\left(\frac{-36 \beta^{2}+18 ß l^{2}}{\mho}\right) w_{1}+\left(\frac{-6 ß l^{2}+2 l^{4}}{\mho}\right) \theta_{1}+\left(\frac{36 \beta^{2}-18 ß l^{2}}{\mho}\right) w_{2}+\left(\frac{6 ß l^{3}}{\mho}\right) \theta_{2} \\
& a_{2}=\left(\frac{6 ß-3 l^{2}}{\mho}\right) w_{1}+\left(\frac{6 ß l-2 l^{3}}{\mho}\right) \theta_{1}+\left(\frac{-6 ß+3 l^{2}}{\mho}\right) w_{2}+\left(\frac{-l^{3}}{\mho}\right) \theta_{2} \\
& a_{3}=\left(\frac{-6 ß+l}{\mho}\right) w_{1}+\left(\frac{6 ß-l}{\mho}\right) w_{2}+\left(\frac{-6 ß l+l^{2}}{\mho}\right) \theta_{2}
\end{aligned}
$$

Changing the transverse deformation $W(x)$ (equation - (14)) in terms of dimension less number $\xi=x / l$ and substituting the values of $a_{i}$ and rearranging.

$$
\begin{aligned}
& W(\xi)=\left(1+\frac{-36 ß^{2}+18 ß l^{2}}{\mho} \xi+\frac{6 ß-3 l^{2}}{\mho} \xi^{2}+\frac{-6 ß+l}{\mho} \xi^{3}\right) w_{1}+\left(\frac{-6 ß l^{2}+2 l^{4}}{\mho} \xi+\right. \\
& \left.\frac{6 ß l-2 l^{3}}{\mho} \xi^{2}\right) \theta_{1}+\left(\frac{36 ß^{2}-18 ß l^{2}}{\mho} \xi+\frac{-6 ß+3 l^{2}}{\mho} \xi^{2}+\frac{6 ß-l}{v} \xi^{3}\right) w_{2}+\left(\frac{6 ß l^{3}}{v} \xi+\frac{-l^{3}}{\mho} \xi^{2}+\frac{-6 ß l+l^{2}}{v} \xi^{3}\right) \theta_{2}
\end{aligned}
$$

$W(\xi)$ can be written in the form 


$$
W(\xi)=\mathcal{N}_{w_{1}} w_{1}+\mathcal{N}_{w_{1}} \theta_{1}+\mathcal{N}_{w_{1}} w_{2}+\mathcal{N}_{w_{1}} \theta_{2}
$$

Where

$$
\begin{aligned}
\mathcal{N}_{w_{1}} & =1+\frac{-36 \beta^{2}+18 ß l^{2}}{\mho} \xi+\frac{6 \beta-3 l^{2}}{\mho} \xi^{2}+\frac{-6 ß+l}{\mho} \xi^{3} \\
\mathcal{N}_{w_{2}} & =\frac{-6 \beta l^{2}+2 l^{4}}{\mho} \xi+\frac{6 \beta l-2 l^{3}}{\mho} \xi^{2} \\
\mathcal{N}_{w_{3}} & =\frac{36 \beta^{2}-18 ß l^{2}}{\mho} \xi+\frac{-6 \beta+3 l^{2}}{\mho} \xi^{2}+\frac{6 \beta-l}{\mho} \xi^{3} \\
\mathcal{N}_{w_{4}} & =\frac{6 \beta l^{3}}{\mho} \xi+\frac{-l^{3}}{\mho} \xi^{2}+\frac{-6 ß l+l^{2}}{\mho} \xi^{3}
\end{aligned}
$$

Substituting values of $a_{i}$ in slope equation $\left(\frac{\partial w}{\partial x}\right)$ due to bending (equation - (16)) in terms of dimension less number $\xi=x / l$ and rearranging.

$$
\begin{aligned}
\Phi(x)-\alpha z^{2}(\Phi(x) & \left.+\frac{d w_{0}(x)}{d x}\right) \\
= & \left(\frac{-36 \beta^{2}+18 ß l^{2}}{\mho}+\frac{12 \beta-6 l^{2}}{\mho} \xi+\frac{-18 ß+3 l}{\mho} \xi^{2}\right) w_{1}+\left(\frac{-6 ß l^{2}+2 l^{4}}{\mho}+\frac{12 ß l-4 l^{3}}{\mho} \xi\right) \theta_{1} \\
+ & \left(\frac{36 \beta^{2}-18 ß l^{2}}{\mho}+\frac{-12 \beta+6 l^{2}}{\mho} \xi+\frac{18 ß-3 l}{\mho} \xi^{2}\right) w_{2}+\left(\frac{6 ß l^{3}}{\mho}+\frac{-2 l^{3}}{\mho} \xi+\frac{-6 ß l+l^{2}}{\mho} \xi^{2}\right) \theta_{2}
\end{aligned}
$$

Hence this slope $\frac{\partial w}{\partial x}=\Phi(x)-\alpha z^{2}\left(\Phi(x)+\frac{d w_{0}(x)}{d x}\right)$ due to bending can also be written as.

$$
\Phi(x)-\alpha z^{2}\left(\Phi(x)+\frac{d w_{0}(x)}{d x}\right)=\mathcal{N}_{\theta_{1}} w_{1}+\mathcal{N}_{\theta_{1}} \theta_{1}+\mathcal{N}_{\theta_{1}} w_{2}+\mathcal{N}_{\theta_{1}} \theta_{2}
$$

Where

$$
\begin{aligned}
& \mathcal{N}_{\theta_{1}}=\frac{-36 \beta^{2}+18 ß l^{2}}{\mho}+\frac{12 \beta-6 l^{2}}{\mho} \xi+\frac{-18 ß+3 l}{\mho} \xi^{2} \\
& \mathcal{N}_{\theta_{2}}=\frac{-6 \beta l^{2}+2 l^{4}}{\mho}+\frac{12 ß l-4 l^{3}}{\mho} \xi \\
& \mathcal{N}_{\theta_{3}}=\frac{36 \beta^{2}-18 ß l^{2}}{\mho}+\frac{-12 \beta+6 l^{2}}{\mho} \xi+\frac{18 ß-3 l}{\mho} \xi^{2} \\
& \mathcal{N}_{\theta_{4}}=\frac{6 \beta l^{3}}{\mho}+\frac{-2 l^{3}}{\mho} \xi+\frac{-6 ß l+l^{2}}{\mho} \xi^{2}
\end{aligned}
$$

Hence with the kinematic equations - (3) and the shape functions we got in equations- (22) and (23), kinematic relations can be expressed as. 


$$
\begin{aligned}
U=(1-\xi) u_{1} & \\
& +z\left(\left(\frac{-36 \aleph^{2}+18 ß l^{2}}{\mho}+\frac{12 ß-6 l^{2}}{\mho} \xi+\frac{-18 ß+3 l}{\mho} \xi^{2}\right) w_{1}\right. \\
& +\left(\frac{-6 ß l^{2}+2 l^{4}}{\mho}+\frac{12 ß l-4 l^{3}}{\mho} \xi\right) \theta_{1} \\
& +\left(\frac{36 ß^{2}-18 ß l^{2}}{\mho}+\frac{-12 ß+6 l^{2}}{\mho} \xi+\frac{18 ß-3 l}{\mho} \xi^{2}\right) w_{2} \\
& \left.+\left(\frac{6 ß l^{3}}{\mho}+\frac{-2 l^{3}}{\mho} \xi+\frac{-6 ß l+l^{2}}{\mho} \xi^{2}\right) \theta_{2}\right)
\end{aligned}
$$

And

$$
\begin{gathered}
W=\left(1+\frac{-36 \beta^{2}+18 ß l^{2}}{\mho} \xi+\frac{6 ß-3 l^{2}}{\mho} \xi^{2}+\frac{-6 ß+l}{\mho} \xi^{3}\right) w_{1}+\left(\frac{-6 ß l^{2}+2 l^{4}}{\mho} \xi+\frac{6 ß l-2 l^{3}}{\mho} \xi^{2}\right) \theta_{1}+ \\
\left(\frac{36 \beta^{2}-18 ß l^{2}}{\mho} \xi+\frac{-6 ß+3 l^{2}}{\mho} \xi^{2}+\frac{6 \beta-l}{\mho} \xi^{3}\right) w_{2}+\left(\frac{6 ß l^{3}}{\mho} \xi+\frac{-l^{3}}{\mho} \xi^{2}+\frac{-6 ß l+l^{2}}{\mho} \xi^{3}\right) \theta_{2}
\end{gathered}
$$

\section{Conclusions:}

For the two node beam element cubic polynomial is assumed for the lateral deformation. By using kinematic assumptions of third order theory as well as the equations that are already derived for the third order theory by J. N. Reddy in 1997, shape functions are derived.

\section{References}

1. J. N. Reddy, “Relationships Between Bending Solutions of Classical and Shear Deformations Beam Theories”, Int. J. Solids Structures Vol. 34, No. 26, pp. 3373-3384, (1997).

2. G. P. Bazeley, Y. K. Cheung, B. M. Irons and O. C. Zienkiewicz, 'Triangular elements in bending-conforming and nonconforming solutions', Proc. Conf: Matrix Methods in Struct. Mech. Mech., Air Force Inst. of Tech., Wright Patterson A. F. Base, Ohio, 1965.

3. Seon M. Han, Haym Benaroya and Timothy Wei, "Dynamics of Transversely Vibrating Beams using four Engineering Theories". Final version. Academic Press (March 22, 1999). Retrieved 2007-04-15.

4. S. P. Timoshenko, "On the correction for shear of the differential equation for transverse vibrations of prismatic bars", Philosophical Magazine (1921) 41,744-746.

5. Timoshenko, S. P. and Woinowsky-Krieger, S., "Theory of Plates and Shells. McGrawHill, Singapore (1970).

6. S. P. Timoshenko and J. N. Goodier, "Theory of Elasticity", Tata McGraw- Hill Educational Private Ltd, 2010. 
7. Klaus-Jürgen Bathe, "Finite element procedures", Second Edition, Massachusetts Institute of Technology, Cambridge, MA 02139, USA, 2014.

8. A. Bazoune and Y.A. Khulief, "Shape functions of Three- dimensional Timoshenko beam element" Journal of Sound and Vibration 259 (2), 473-480 (2003).

9. G. Jemielita, Techniczna teoria plyt srednieej grubbosci, (Technical theory of plates with moderate thickness), Rozprawy Inzynierskie (Engineering Transactions), Polska Akademia Nauk 23(3), (1975) 483-499.

10. M. Levinson, “A New Rectangular Beam Theory” Journal of Sound and Vibration (1981) 74(1), 81-87.

11. Bickford, W. B., “A consistent higher order beam theory”. Developments Theoretical and Applied Mechanics 11,137-150 (1982).

12. J. N. Reddy, “A Simple Higher-Order Theory for Laminated Composite Plates" Journal of Applied Mechanics December 1984, Vol. 51/745.

13. J.N. Reddy, Mechanics of Laminated Composite Plates: Theory and Analysis, CRC Press, Boca Raton, FL, (1997).

14. Ambartsumyan, S. A., "On the theory of bending plates", Izv Otd Tech Nauk AN SSSR 5 (1958) 69-77.

15. Kruszewski, E. T., Effect of transverse shear and rotatory inertia on the natural frequency of a uniform beam, NACATN (1909).

16. Akavci, S. S., Buckling and free vibration analysis of symmetric and anti-symmetric laminated composite plates on an elastic foundation, Journal of Reinforced Plastics and Composites 26 (18) (2007) 1907-1 919.

17. Reddy, J. N., A general non-linear third order theory of plates with moderate thickness, International Journal of Non-linear Mechanics 25 (6) (1990) 677-686.

18. Touratier, M., An efficient standard plate theory, International Journal of Engineering Science 29 (8) (1991) 901-916.

19. Soldatos, K. P., A transverse shear deformation theory for homogeneous monoclinic plates, Acta Mechanica 94 (1992) 195-200.

20. Karama, M., Afaq, K. S., Mistou, S., Mechanical behavior of laminated composite beam by new multi-layered laminated composite structures model with transverse shear stress continuity, International Journal of Solids and Structures, 40 (2003) 1525-1546. 